\title{
Q-CURVATURE AND POINCARÉ METRICS
}

\author{
Charles Fefferman and C. Robin Graham
}

\section{Introduction}

This article presents a new definition of Branson's $Q$-curvature in even dimensional conformal geometry. The $Q$-curvature is a generalization of the scalar curvature in dimension 2: it satisfies an analogous transformation law under conformal rescalings of the metric and on conformally flat manifolds its integral is a multiple of the Euler characteristic. Our approach is motivated by the recent work [GZ]; we derive the $Q$-curvature as a coefficient in the asymptotic expansion of the formal solution of a boundary problem at infinity for the Laplacian in the Poincaré metric associated to the conformal structure. This gives an easy proof of the result of [GZ] that the log coefficient in the volume expansion of a Poincaré metric is a multiple of the integral of the $Q$-curvature, and leads to a definition of a non-local version of the $Q$-curvature in odd dimensions.

The $Q$-curvature is intimately connected with a family of conformally invariant differential operators generalizing the conformal Laplacian $\Delta+\frac{n-2}{4(n-1)} R$, for which the scalar curvature arises as the zeroth order term. (Our sign convention is such that $\Delta$ is a positive operator.) The next operator in the family was discovered by Paneitz $[\mathrm{Pa}]$ and has the same principal part as $\Delta^{2}$. Branson and Ørsted [BØ] observed that the zeroth order term of Paneitz' operator gives rise to the quantity

$$
Q=\left(\Delta R+R^{2}-3|\operatorname{Ric}|^{2}\right) / 6
$$

in 4 dimensions with a conformal transformation law similar to that of scalar curvature in dimension 2. This $Q$-curvature in dimension 4 has been the focus of tremendous activity in recent years leading to great advances in our understanding of 4 dimensional conformal geometry; see [CY] for a survey of some of this work. The full family of "conformally invariant powers of the Laplacian" was derived in [GJMS], and Branson [B] formulated the definition of the $Q$-curvature in general even dimensions using these operators. However, this general definition involves an analytic continuation in the dimension and the higher-dimensional $Q$-curvature has remained a rather mysterious object.

The work [GZ] shows that the conformally invariant powers of the Laplacian and the $Q$-curvature arise naturally in scattering theory for Poincaré metrics associated to the conformal structure. This connection can be thought of as

Received October 24, 2001.

The work of the first author was partially supported by NSF grant DMS-0070692. 
providing a different definition of the $Q$-curvature, in which the analytic continuation in the dimension is replaced by an analytic continuation in a spectral parameter with the dimension fixed. Conceptually this seems an improvement, but the definition is still indirect. Our contribution is to provide a direct definition in the context of the Poincaré metric.

The Poincaré metrics involved are asymptotically Einstein metrics whose conformal infinity is the given conformal structure. The model is hyperbolic space $\mathbb{H}^{n+1}=\left\{(x, y) \in \mathbb{R}_{+} \times \mathbb{R}^{n}\right\}$, with metric $g=x^{-2}\left(d x^{2}+\sum d y_{i}^{2}\right)$. On $\mathbb{H}^{n+1}$, the function $U=\log x$ satisfies $\Delta_{g} U=n$. In $\S 3$, we show that if $g$ is a formal Poincaré metric associated to the conformal structure, the equation $\Delta_{g} U=n$ has a formal solution asymptotic to $\log x+O(x)$, where $x$ is a defining function for the boundary at infinity associated to a metric in the conformal class. The $Q$-curvature is a multiple of the coefficient of $x^{n} \log x$ in the expansion of $U$. The conformal transformation law for $Q$ follows immediately from the characterization given in [GZ] of the conformally invariant $(n / 2)^{\text {th }}$ power of the Laplacian $P_{n / 2}$ as the coefficient of $x^{n} \log x$ in the expansion of a formal solution of $\Delta_{g} u=0$ with prescribed boundary value. The result that the log term coefficient in the volume expansion is the integral of $Q$ follows by an application of Green's identity.

In $\S 4$, we study the equation $\Delta_{g} U=n$ globally. We show that in any dimension there is a unique global solution with the prescribed leading asymptotics, given by the formula $U=-\left.\frac{d}{d s} \mathcal{P}(s) 1\right|_{s=n}$, where $\mathcal{P}(s)$ is the family of Poisson operators for $\Delta_{g}-s(n-s)$. If $n$ is odd, there is no $x^{n} \log x$ term in the expansion of $U$, but the $x^{n}$ coefficient provides a non-local analogue of $Q$. This odd-dimensional version satisfies a conformal transformation law just like the one in even dimensions, in which $P_{n / 2}$ is replaced by a pseudodifferential operator acting invariantly on conformal densities with principal part the same as that of $\Delta^{n / 2}$; namely a multiple of $S(n)$, where $S(s)$ is the scattering matrix for $\Delta_{g}-s(n-s)$. The same Green's identity argument as in even dimensions shows that in odd dimensions, the renormalized volume itself is a multiple of the integral of the odd-dimensional $Q$. One important distinction between even and odd dimensions, though, is that in odd dimensions both $Q$ and the operator $S(s)$ are not determined solely by the conformal structure on the boundary at infinity, but also depend on the extension of the formal Poincaré metric to a metric on the interior. This dependence could be a limitation for their use in the study of conformal geometry.

We include in $\S 3$ a different proof of another result of [GZ]: the self-adjointness of the conformal powers of the Laplacian of [GJMS]. In [GZ] this was shown to follow by analytic continuation from the self-adjointness of the scattering matrix, of which the conformal powers of the Laplacian arise as residues. We show that a renormalized integration by parts identity giving self-adjointness of the scattering matrix can be formulated directly at the limiting value and gives a proof based purely on formal asymptotics. 


\section{Background}

In this section we discuss background material about Poincaré metrics, conformally invariant powers of the Laplacian, $Q$-curvature, and volume renormalization which we will need later.

Let $M$ be a manifold of dimension $n>2$ with a conformal structure [h]. Any two metrics $h, \hat{h} \in[h]$ are conformally related: $\hat{h}=e^{2 \Upsilon} h$ for some $\Upsilon \in C^{\infty}(M)$. The conformal structure is equivalent to the specification of the metric bundle of $[h]$. This is the ray bundle $\mathcal{G} \subset S^{2} T^{*} M$ of multiples of the metric: the fiber of $\mathcal{G}$ over $p \in M$ is $\left\{t^{2} h(p): t>0\right\}$. The space of conformal densities of weight $w \in \mathbb{C}$ is

$$
\mathcal{E}(w)=C^{\infty}\left(M ; \mathcal{G}^{-\frac{w}{2}}\right),
$$

where by abuse of notation we have denoted by $\mathcal{G}$ also the line bundle associated to the ray bundle defined above. A choice of representative $h$ for the conformal structure induces an identification $\mathcal{E}(w) \simeq C^{\infty}(M)$; if $\hat{h}=e^{2 \Upsilon} h$ then the corresponding elements of $C^{\infty}(M)$ transform by $\hat{f}=e^{w \Upsilon} f$.

Our paper $[\mathrm{FG}]$ introduced two formal metrics in higher dimensions which can be associated to the conformal structure $(M,[h])$. The relevant one here is the Poincaré metric. If $X$ is an $n+1$-manifold with $\partial X=M$ and $x$ is a defining function of $\partial X=M$ in $X$ :

$$
\left.x\right|_{X} ^{\circ}>0,\left.\quad x\right|_{\partial X}=0,\left.\quad d x\right|_{\partial X} \neq 0,
$$

we say that a metric $g$ on $\stackrel{\circ}{X}$ is conformally compact with conformal infinity $[h]$ if

$$
g=\frac{\bar{g}}{x^{2}},\left.\quad \bar{g}\right|_{T \partial X} \in[h]
$$

where $\bar{g}$ is a smooth metric on $X$. A conformally compact metric is said to be asymptotically hyperbolic if its sectional curvatures approach -1 at $\partial X$; this is equivalent to $|d x|_{\bar{g}}=1$ on $\partial X$. It was shown in [GL] that if $g$ is an asymptotically hyperbolic metric on $X$, then a choice of metric $h$ in the conformal class on $M$ uniquely determines a defining function $x$ near $\partial X$ and an identification of a neighborhood of $\partial X$ with $M \times[0, \epsilon)$ such that $g$ has the normal form

$$
g=x^{-2}\left(h_{x}+d x^{2}\right), \quad h_{0}=h,
$$

where $h_{x}$ is a 1-parameter family of metrics on $M$. We will say that $x$ is the defining function associated to $h$.

The Poincaré metric of $(M,[h])$ is conformally compact and is an asymptotic solution of the Einstein equation $\operatorname{Ric}(g)=-n g$. It is easy to see that any such metric is asymptotically hyperbolic. In order to formally solve this equation, we identify a neighborhood of $M$ in $X$ with $M \times[0, \epsilon)$ and consider metrics of the form (2.1). The equation $\operatorname{Ric}(g)+n g=0$ can be calculated directly in terms of $h_{x}$ and the formal asymptotics of solutions studied; see [G]. If $n$ is odd, then there is a unique formal smooth solution $h_{x}$ to $\operatorname{Ric}(g)+n g=O\left(x^{\infty}\right)$ which is even in $x$. If $n$ is even, the condition $\operatorname{Ric}(g)+n g=O\left(x^{n-2}\right)$ uniquely 
determines $h_{x} \bmod O\left(x^{n}\right)$, and this $h_{x}$ is even in $x\left(\bmod O\left(x^{n}\right)\right)$. Although in general smooth solutions do not exist to higher orders for $n$ even, the condition $\operatorname{tr}_{g}(\operatorname{Ric}(g)+n g)=O\left(x^{n+2}\right)$ can be satisfied and uniquely determines the $h$-trace of the $x^{n}$ coefficient in $h_{x}$. The indicated Taylor coefficients of $h_{x}$ are determined inductively from the equation and are given by polynomial formulae in terms of $h$, its inverse, and its curvature tensor and covariant derivatives thereof.

Since any asymptotically hyperbolic metric can be put uniquely in the form (2.1) upon choosing $h$, it follows that the equivalence class of the solution $g$ up to diffeomorphism and up to terms vanishing to the indicated orders is uniquely determined by the conformal structure. This equivalence class is called the formal Poincaré metric associated to $[h]$. When $n$ is even, the higher order terms in $h_{x}$ are not determined and will not play a role in this paper. Similarly, it is not crucial that the metric be smooth to all orders-honest Einstein metrics will have log terms in their expansions, but these also occur to orders that do not affect the considerations here. When $n$ is odd, a number of our results hold without the assumption that $h_{x}$ is even beyond the order forced by the Einstein equation. However, for simplicity in statements below, we shall restrict attention to $h_{x}$ which are smooth and even in $x$ to all orders. If $X$ is a manifold with $\partial X=M$, we shall call a Poincaré metric associated to $[h]$ any metric on $X$ whose restriction to a collar neighborhood of $M$ is such a formal Poincaré metric.

The conformally invariant powers of the Laplacian are a family $P_{k}, k \in \mathbb{N}$ and $k \leq n / 2$ if $n$ is even, of natural differential operators on $n$-dimensional Riemannian manifolds; $P_{k}$ has the same principal part as $\Delta^{k}$ and equals $\Delta^{k}$ if $h$ is flat. $P_{k}$ is conformally invariant in the sense that it defines an operator $P_{k}: \mathcal{E}(-n / 2+k) \rightarrow \mathcal{E}(-n / 2-k)$ on conformal densities independent of the choice of conformal representative, which is equivalent to the statement that under conformal rescaling $\hat{h}=e^{2 \Upsilon} h, P_{k}$ transforms by

$$
\widehat{P}_{k}=e^{(-n / 2-k) \Upsilon} P_{k} e^{(n / 2-k) \Upsilon} .
$$

The operators $P_{k}$ were constructed in [GJMS] using the ambient metric of [FG]. In [GZ] it was shown that the derivation can be reformulated in terms of the Poincaré metric by consideration of the formal asymptotics of solutions of

$$
\left(\Delta_{g}-s(n-s)\right) u=O\left(x^{\infty}\right) .
$$

If $g$ is a Poincaré metric for $(M,[h])$ and $x, \hat{x}$ the are the defining functions associated to the representative metrics $h, \hat{h}$, then $\hat{x}=e^{\Upsilon} x+O\left(x^{2}\right)$. It follows that if $f \in C^{\infty}(M)$ represents a section of $\mathcal{E}(w)$, then it is a conformally invariant statement to require that a function $u$ on $X$ be asymptotic to $x^{-w} f$. The operator $P_{k}$ is derived in [GZ] as the obstruction to the existence of a smooth solution of (2.2) for $s=n / 2+k$ with $u$ having prescribed leading term $x^{n / 2-k} f$ :

Proposition 2.1. ([GZ, Propositions 4.2, 4.3]) Let $g$ be a Poincaré metric associated to $(M,[h])$ and let $f \in C^{\infty}(M)$. Let $k \in \mathbb{N}$ with $k \leq n / 2$ if $n$ is even. 
There is a solution of (2.2) for $s=n / 2+k$ of the form

$$
u=x^{n / 2-k}\left(F+H x^{2 k} \log x\right)
$$

with $F, H \in C^{\infty}(X)$ and with $\left.F\right|_{M}=f$. The functions $F \bmod O\left(x^{2 k}\right)$ and $\left.H\right|_{M}$ are formally determined by $h$, and

$$
\left.H\right|_{M}=-2 c_{k} P_{k} f, \quad c_{k}=(-1)^{k}\left[2^{2 k} k !(k-1) !\right]^{-1},
$$

where $P_{k}$ is the conformally invariant operator of [GJMS].

Branson's definition of the $Q$-curvature is in terms of the zeroth order terms of the operators $P_{k}$. Note that if $n$ is even, then $P_{n / 2}$ is invariantly defined from $\mathcal{E}(0)=C^{\infty}(M)$ to $\mathcal{E}(-n)$. Since the constant function $1 \in \mathcal{E}(0)$ has a smooth extension annihilated by $\Delta_{g}$, we have $P_{n / 2} 1=0$. Therefore $P_{n / 2}$ has no constant term in dimension $n$. However, if we fix $k \in \mathbb{N}$ and denote by $P_{k}^{n}$ the operator $P_{k}$ in dimension $n$, then $P_{k}^{n}$ has coefficients given by universal formulae in curvature and its derivatives which are rational in $n$, and the zeroth order term of $P_{k}^{n}$ is of the form $(n / 2-k) Q_{k}^{n}$ for a scalar Riemannian invariant $Q_{k}^{n}$ with coefficients rational in $n$ and regular at $n=2 k$. The $Q$-curvature in even dimension $n$ is then defined as $Q=Q_{n / 2}^{n}$. An analytic continuation argument in the dimension using the conformal transformation law of the operators $P_{k}$ (see [B]) yields the invariance property of $Q$ : if once again $\hat{h}=e^{2 \Upsilon} h$, then

$$
e^{n \Upsilon} \widehat{Q}=Q+P_{n / 2} \Upsilon
$$

From this and the facts that $P_{n / 2}$ is self-adjoint and $P_{n / 2} 1=0$, it follows that $\int_{M} Q d v_{h}$ is independent of the choice of $h$ in the conformal class.

We have learned that Gover-Peterson [GP] have found a construction of the operators $P_{k}$ and $Q$ using the conformal tractor calculus.

Let $(X, g)$ be a Poincaré metric associated to $(M,[h])$. The AdS/CFT correspondence in physics has given rise to interesting invariants which arise in the asymptotics of the volume function on $X$ - see [W], [HS], [GW], [G]. To define these invariants, observe that if $h$ is a metric in the conformal class and $x$ is the associated defining function, then (2.1) implies that near $\partial X$, the volume form of $g$ takes the form $d v_{g}=x^{-n-1} d v_{h_{x}} d x$, where

$$
d v_{h_{x}}=\left(1+v^{(2)} x^{2}+(\text { even powers })+\ldots\right) d v_{h}
$$


and the $v^{(2 j)}$ are smooth functions on $M$ determined by $h$ (for $1 \leq j \leq n / 2$ if $n$ is even). Integration shows that

$$
\operatorname{vol}_{g}(\{x>\epsilon\})=c_{0} \epsilon^{-n}+c_{2} \epsilon^{-n+2}+\cdots+c_{n-1} \epsilon^{-1}+V+o(1)
$$

for $n$ odd,

$$
\operatorname{vol}_{g}(\{x>\epsilon\})=c_{0} \epsilon^{-n}+c_{2} \epsilon^{-n+2}+\cdots+c_{n-2} \epsilon^{-2}+L \log (1 / \epsilon)+V+o(1)
$$

for $n$ even.

It can be shown (see [G]) that $V$ is independent of the conformal representative $h$ on the boundary at infinity when $n$ is odd, and $L$ is independent of the conformal representative when $n$ is even. Graham-Zworski [GZ] have recently identified $L$ as the integral of the $Q$-curvature: $L=2 c_{n / 2} \int_{M} Q d v_{h}$, where $c_{n / 2}$ is as in (2.4). We shall give another proof of this result below. Anderson [A1] has identified $V$ when $n=3$. In an appendix to [PP], Epstein shows that for conformally compact hyperbolic manifolds, the invariants $L$ for $n$ even and $V$ for $n$ odd are each multiples of the Euler characteristic $\chi(X)$. The dependence of $V$ on the choice of $h$ for $n$ even is the so-called holographic anomaly - see [HS],[G].

\section{Formal Considerations}

The considerations of this section are all formal near the boundary, so we shall take $X=M \times[0,1)$.

Theorem 3.1. Suppose $n$ is even and let $(X, g)$ be a Poincaré metric associated to $(M,[h])$. Choose a representative metric $h$ with special defining function $x$. There is a unique solution $U \bmod O\left(x^{n}\right)$ to

$$
\Delta_{g} U=n+O\left(x^{n+1} \log x\right)
$$

of the form

$$
U=\log x+A+B x^{n} \log x+O\left(x^{n}\right),
$$

with

$$
A, B \in C^{\infty}(X),\left.\quad A\right|_{M}=0 .
$$

Also, $A \bmod O\left(x^{n}\right)$ and $\left.B\right|_{M}$ are formally determined by $h$.

Proof. Write $g$ in the form (2.1). A straightforward calculation shows that

$$
\Delta_{g}=-\left(x \partial_{x}\right)^{2}+\left(n-\frac{x}{2} \operatorname{tr}_{h_{x}} h_{x}^{\prime}\right) x \partial_{x}+x^{2} \Delta_{h_{x}},
$$

where $h_{x}^{\prime}=\partial_{x} h_{x}$. Therefore the equation $\Delta_{g} U=n$ is equivalent to

$$
\Delta_{g}(U-\log x)=\frac{x}{2} \operatorname{tr}_{h_{x}} h_{x}^{\prime} .
$$


In order to construct a solution to this equation, observe that (3.3) implies that if $a_{j} \in C^{\infty}(M)$, then

$$
\Delta_{g}\left(a_{j} x^{j}\right)=j(n-j) a_{j} x^{j}+O\left(x^{j+1}\right) .
$$

Since $x \operatorname{tr}_{h_{x}} h_{x}^{\prime} \in x^{2} C^{\infty}(X)$ and is formally determined by $h \bmod O\left(x^{n+1}\right)$, we can take $a_{0}=a_{1}=0$ and then inductively determine $a_{j}$ uniquely for $1<j<n$ so that $A=\sum_{j=2}^{n-1} a_{j} x^{j}$ solves

$$
\Delta_{g} A=\frac{x}{2} \operatorname{tr}_{h_{x}} h_{x}^{\prime}+x^{n} E,
$$

where $E \in C^{\infty}(X)$ and $\left.E\right|_{M}$ is formally determined by $h$. The $x^{n} E$ error cannot be removed by a term of the form $a_{n} x^{n}$, but it can by a term of the form $B x^{n} \log x$ since

$$
\Delta_{g}\left(B x^{n} \log x\right)=-n B x^{n}+O\left(x^{n+1} \log x\right) .
$$

Also $\left.B\right|_{M}$ is formally determined by $h$ since we must take $-\left.n B\right|_{M}=\left.E\right|_{M}$.

We observe that since $h_{x}$ is an even function of $x$, it follows from the inductive construction above that $A \bmod O\left(x^{n}\right)$ is also even. An argument similar to the above shows that when $n$ is odd, there is a unique formally determined solution $U \bmod O\left(x^{\infty}\right)$ to $\Delta U=n+O\left(x^{\infty}\right)$ of the form $U=\log x+A$ with $A \in C^{\infty}(X),\left.A\right|_{M}=0, A \bmod O\left(x^{\infty}\right)$ even in $x$. However, as we shall see in the next section, for $n$ odd the interesting term analogous to $B x^{n} \log x$ is globally determined. When $n$ is even, the solution $U$ of Theorem 3.1 can be continued to higher order, but is no longer formally determined by $h$.

The function $\left.B\right|_{M}$ in Theorem 3.1 is an invariant of the metric $h$. We shall show in Theorem 4.1 that

$$
\left.B\right|_{M}=-2 c_{n / 2} Q
$$

where $Q$ is Branson's $Q$-curvature, so that the construction of Theorem 3.1 provides a characterization of $Q$. For now we take (3.4) as a definition and show how to deduce the transformation law (2.5).

Suppose we choose a different metric $\hat{h}=e^{2 \Upsilon} h$ in Theorem 3.1. Then $\hat{x}=e^{\Upsilon} x$ $\bmod O\left(x^{2}\right)$ so that $\log \hat{x}=\log x+\Upsilon+O(x)$. It follows that $\Delta_{g}(\hat{U}-U)=$ $O\left(x^{n+1} \log x\right)$ and $\hat{U}-U=F+H x^{n} \log x+O\left(x^{n}\right)$, where $F, H \in C^{\infty}(X)$ and $\left.F\right|_{M}=\Upsilon$. By Proposition 2.1, this problem solved by $\hat{U}-U$ is precisely the one giving rise to the invariant operator $P_{n / 2}$, so we deduce that

$$
\left.H\right|_{M}=-2 c_{n / 2} P_{n / 2} \Upsilon \text {. }
$$

However, subtracting the expansions (3.2) for $\hat{U}$ and $U$ shows that

$$
\left.H\right|_{M}=\left.e^{n \Upsilon} \hat{B}\right|_{M}-\left.B\right|_{M},
$$

giving (2.5).

We next show how to use the function $U$ to give a simple proof of the following theorem of [GZ]. Recall that $L$ denotes the coefficient of the logarithmic term in $(2.7)$. 
Theorem 3.2. If $n$ is even, then

$$
L=2 c_{n / 2} \int_{M} Q d v_{h} .
$$

Proof. Let $x_{0}>0$ be small. Recalling (2.1), Green's identity gives

$$
\int_{\epsilon<x<x_{0}} \Delta_{g} U d v_{g}=\epsilon^{1-n} \int_{x=\epsilon} \partial_{x} U d v_{h_{\epsilon}}-x_{0}^{1-n} \int_{x=x_{0}} \partial_{x} U d v_{h_{x_{0}}},
$$

which together with (3.1) yields

$$
n \operatorname{vol}_{g}\left\{\epsilon<x<x_{0}\right\}=\left.\epsilon^{1-n} \int_{M} \partial_{x} U\right|_{x=\epsilon} d v_{h_{\epsilon}}+O(1) .
$$

By definition, the coefficient of $\log \epsilon$ in the expansion of the left hand side is $-n L$. Differentiating (3.2) and using (2.6) shows that the coefficient of $\log \epsilon$ on the right hand side is $n \int_{M} B d v_{h}$, so the result follows from (3.4).

The fact that the conformally invariant operators $P_{k}$ of [GJMS] are selfadjoint was proved in [GZ] by analytic continuation in the spectral parameter, using the self-adjointness on the real axis of the scattering matrix. One way that the scattering matrix can be proved to be self-adjoint is via a renormalized integration by parts identity (Proposition 3.3 of [GZ]) originally discussed for hyperbolic space in $[\mathrm{W}]$. In the next proposition, we show that a variant of this identity gives self-adjointness of the $P_{k}$ directly.

Proposition 3.3. Let $(X, g)$ be a Poincaré metric associated to $(M,[h])$, let $k \in \mathbb{N}$ with $k \leq n / 2$ if $n$ is even, and set $s=n / 2+k / 2$. Let $f_{1}, f_{2} \in C^{\infty}(M)$ and let $u_{1}, u_{2}$ denote the corresponding solutions of (2.2) given by Proposition 2.1. Then for fixed small $x_{0}>0$,

$$
\begin{aligned}
\operatorname{lp} \int_{\epsilon<x<x_{0}} & {\left[\left\langle d u_{1}, d u_{2}\right\rangle-s(n-s) u_{1} \overline{u_{2}}\right] d v_{g} } \\
& =2 n c_{k} \int_{M} f_{1} \overline{P_{k} f_{2}} d v_{h}=2 n c_{k} \int_{M} \overline{f_{2}} P_{k} f_{1} d v_{h},
\end{aligned}
$$

where $\operatorname{lp}$ denotes the coefficient of $\log \epsilon$ in the asymptotic expansion of the integral as $\epsilon \rightarrow 0$. In particular, $P_{k}$ is self-adjoint.

Proof. With $g$ in the form (2.1), Green's identity gives

$$
\begin{aligned}
& \int_{\epsilon<x<x_{0}}\left[\left\langle d u_{1}, d u_{2}\right\rangle-s(n-s) u_{1} \overline{u_{2}}\right] d v_{g} \\
& =\int_{\epsilon<x<x_{0}} u_{1}(\Delta-s(n-s)) \overline{u_{2}} d v_{g}+x_{0}^{1-n} \int_{x=x_{0}} u_{1} \partial_{x} \overline{u_{2}} d v_{h_{x_{0}}} \\
& =-\epsilon^{1-n} \int_{x=\epsilon} u_{1} \partial_{x} \overline{u_{2}} d v_{h_{\epsilon}}+O(1) .
\end{aligned}
$$


Substituting (2.3) and (2.6) and expanding shows that the coefficient of $\log \epsilon$ in the expansion of this expression is

$$
-\int_{M}\left[(n / 2+k) F_{1} \overline{H_{2}}+(n / 2-k) H_{1} \overline{F_{2}}\right] d v_{h} .
$$

By symmetry in $u_{1}, u_{2}$, we deduce that this equals

$$
-n \int_{M} F_{1} \overline{H_{2}} d v_{h}=-n \int_{M} H_{1} \overline{F_{2}} d v_{h}
$$

which gives the desired result by (2.4).

\section{Global Considerations}

In this section, let $X$ be a compact $n+1$-manifold with $\partial X=M$. (If there is no such $X$, one can take instead $X=M \times[0,1]$, for which $\partial X=M \sqcup M$.) Let $g$ be a Poincaré metric on $X$ with conformal infinity $(M,[h])$, and choose a metric $h$ in the conformal class with associated defining function $x$. The main theorem of this section is the following:

Theorem 4.1. There is a unique function $U \in C^{\infty}(\stackrel{\circ}{X})$ solving

$$
\Delta_{g} U=n
$$

and with the asymptotics

$$
U= \begin{cases}\log x+A+B x^{n} \log x & \text { for } n \text { even } \\ \log x+A+B x^{n} & \text { for } n \text { odd }\end{cases}
$$

where $A, B \in C^{\infty}(X)$ are even $\bmod O\left(x^{\infty}\right)$ and $\left.A\right|_{M}=0$. Moreover, if $n$ is even, we have

$$
\left.B\right|_{M}=-2 c_{n / 2} Q .
$$

Our analysis of this global problem depends on results from [GZ] on the asymptotic Dirichlet problem at infinity for the operator $\Delta_{g}-s(n-s)$, which we briefly review. Mazzeo and Mazzeo-Melrose [Ma1], [MM], [Ma2] showed that the spectrum of $\Delta_{g}$ is of the form $\Sigma \cup\left[(n / 2)^{2}, \infty\right)$, where $\Sigma \subset\left(0,(n / 2)^{2}\right)$ is the finite set of $L^{2}$ eigenvalues. Using fundamental results of [MM] concerning the existence and properties of the resolvent, in [GZ] (see also [JS]), it was shown that there is a meromorphic family of Poisson operators

$$
\mathcal{P}(s): \mathcal{E}(s-n) \longrightarrow C^{\infty}(\stackrel{\circ}{X})
$$

for $\operatorname{Re} s>n / 2$, with poles only for $s$ such that $s(n-s) \in \Sigma$, with the characterizing properties:

$$
\begin{aligned}
& \left(\Delta_{g}-s(n-s)\right) \mathcal{P}(s) f=0, \\
& \mathcal{P}(s) f=x^{n-s} F+x^{s} G \quad \text { if } s \notin n / 2+\mathbb{N} \\
& \mathcal{P}(s) f=x^{n / 2-k} F+H x^{n / 2+k} \log x \quad \text { if } s=n / 2+k, k \in \mathbb{N},
\end{aligned}
$$

for $F, G, H \in C^{\infty}(X)$ such that $\left.F\right|_{M}=f$. If $2 s-n$ is not an odd integer, then $F, G, H \bmod O\left(x^{\infty}\right)$ are uniquely determined and are even. If $2 s-n$ is an odd 
integer, then $F, G \bmod O\left(x^{\infty}\right)$ may be chosen to be even and are then uniquely determined.

If $s=n / 2+k$ for $k \in \mathbb{N}$ satisfying $k \leq n / 2$ if $n$ is even, then $\left.H\right|_{M}$ is locally determined by $f$ and $h$. In fact, by Proposition 2.1, in this case we have $\left.H\right|_{M}=-2 c_{k} P_{k} f$ where $P_{k}$ is the conformally invariant operator on $M$. However, if $s \notin n / 2+\mathbb{N}$, then $\left.G\right|_{M}$ is globally determined by $f$ and $g$ and defines the scattering matrix:

$$
S(s) f=\left.G\right|_{M} .
$$

It can be shown ([Me], $[\mathrm{JS}],[\mathrm{GZ}])$ that

$$
S(s): \mathcal{E}(s-n) \longrightarrow \mathcal{E}(-s)
$$

is a meromorphic family of pseudodifferential operators on $M$ for $\operatorname{Re} s>n / 2$, of order $2 \operatorname{Re} s-n$, invariantly defined on densities, with poles only for $s(n-s) \in \Sigma$ and $s \in n / 2+\mathbb{N}$, and with principal symbol

$$
\sigma(S(s))=2^{n-2 s} \frac{\Gamma(n / 2-s)}{\Gamma(s-n / 2)} \sigma\left(\Delta_{h}^{s-n / 2}\right) .
$$

The full symbol of $S(s)$ is determined by $g \bmod O\left(x^{\infty}\right)$, and therefore by $h$ if $n$ is odd (and to the appropriate order if $n$ is even). Theorem 1 of [GZ] shows that if $k \in \mathbb{N}$ and $k \leq n / 2$ for $n$ even, and $(n / 2+k)(n / 2-k) \notin \Sigma$, then the pole of $S(s)$ at $s=n / 2+k$ is simple and

$$
\operatorname{Res}_{s=n / 2+k} S(s)=-c_{k} P_{k} .
$$

Here we have used the metric $h$ to trivialize the density bundles and so regard all $S(s)$ as operators on $C^{\infty}(M)$.

Observe that $\mathcal{P}(s)$ is always regular at $s=n, \mathcal{P}(n)$ acts invariantly on $C^{\infty}(M)=\mathcal{E}(0)$, and we have $\mathcal{P}(n) 1=1$. In particular, from the expansion we obtain $P_{n / 2} 1=0$ for $n$ even and $S(n) 1=0$ for $n$ odd. If $n$ is even, (4.5) then shows that $S(s) 1$ extends holomorphically across $s=n$, and if we set $S(n) 1=\lim _{s \rightarrow n} S(s) 1$, then Theorem 2 of [GZ] states that

$$
S(n) 1=c_{n / 2} Q .
$$

Still using $h$ to trivialize the density bundles, consider the expansion

$$
\mathcal{P}(s) 1=x^{n-s} F_{s}+x^{s} G_{s}
$$

for $s$ near $n$, with $F_{s}, G_{s}$ mod $O\left(x^{\infty}\right)$ even as described above. Of course, such an expansion holds also for $s=n$, and for $n$ odd it is clear that $F_{n}=1, G_{n}=$ $0 \bmod O\left(x^{\infty}\right)$. However, if $n$ is even, the expansion alone does not uniquely determine $F_{n}, G_{n} \bmod O\left(x^{\infty}\right)$. The construction of the Poisson operators in [GZ] shows that $F_{s}, G_{s} \in C^{\infty}(X)$ may be chosen to depend holomorphically on $s$ across $s=n$, which fixes this ambiguity in $F_{n}, G_{n} \bmod O\left(x^{\infty}\right)$. The definition (4.3) of the scattering matrix together with (4.6) give $\left.G_{n}\right|_{M}=c_{n / 2} Q$, so the condition $\mathcal{P}(n) 1=1$ forces $F_{n}=1-c_{n / 2} Q x^{n}+O\left(x^{n+1}\right)$. (Actually, the proof of (4.6) in [GZ] is by the reverse of this logic: the $x^{n}$ coefficient of $F_{n}$ is 
evaluated first by taking the limit of the formal solutions $F_{s} \bmod O\left(x^{\infty}\right)$. By arguing along these lines, it is possible to give a purely formal proof of (4.2).)

Proof of Theorem 4.1. Consider the holomorphic family of functions $\mathcal{P}(s) 1 \in$ $C^{\infty}(\stackrel{\circ}{X})$ for $s$ near $n$. We have $\left[\Delta_{g}-s(n-s)\right] \mathcal{P}(s) 1=0$. Differentiation with respect to $s$ at $s=n$ gives $\Delta_{g} U=n$, where

$$
U=-\left.\frac{d}{d s} \mathcal{P}(s) 1\right|_{s=n} .
$$

Differentiation of (4.7) yields

$$
U=F_{n} \log x-F_{n}^{\prime}-G_{n} x^{n} \log x-x^{n} G_{n}^{\prime},
$$

where ${ }^{\prime}=d / d s$. Substituting the values derived above (and recalling that $\left.F_{s}\right|_{M}=1$ so that $\left.\left.F_{n}^{\prime}\right|_{M}=0\right)$ gives the stated asymptotics of $U$.

Uniqueness follows from the facts that the asymptotics of $U$ up to the $x^{n}$ term are formally determined and that there are no $L^{2} \Delta_{g}$-harmonic functions.

We remark that on a general asymptotically hyperbolic manifold, the same argument produces a unique solution of $\Delta_{g} U=n$ with leading asymptotics $\log x+O(x)$.

If $n$ is odd, then $\left.B\right|_{M} \in C^{\infty}(M)$ is determined by the choice of Poincaré metric $g$ and representative metric $h$, and the construction above shows that $\left.B\right|_{M}=-\left.\frac{d}{d s} S(s) 1\right|_{s=n}$. We define an analogue of the $Q$-curvature for $n$ odd by

$$
Q=\left.k_{n} B\right|_{M}=-\left.k_{n} \frac{d}{d s} S(s) 1\right|_{s=n}, \quad k_{n}=2^{n} \frac{\Gamma(n / 2)}{\Gamma(-n / 2)} .
$$

This quantity is globally determined and depends in general on the extension of the formal Poincaré metric to a metric on $X$. It is tempting to try to normalize this extension by requiring that it be exactly Einstein, especially in light of the significant recent progress on this existence question ([A2]). However, an Einstein extension might not exist and might not in general be unique.

For the odd dimensional version of $Q$, we have the following analogue of (2.5):

Proposition 4.2. If $n$ is odd, then under a conformal change $\hat{h}=e^{2 \Upsilon} h, Q$ satisfies the transformation law:

$$
e^{n \Upsilon} \widehat{Q}=Q+k_{n} S(n) \Upsilon .
$$

Proof. As in the proof of (2.5) given in $\S 3$, the difference $\hat{U}-U$ is a solution of $\Delta_{g}(\hat{U}-U)=0$ with $\left.(\hat{U}-U)\right|_{M}=\Upsilon$. By the definition of the scattering matrix, the $x^{n}$ coefficient in the expansion of $\hat{U}-U$ is $S(n) \Upsilon$, which gives $\left.e^{n \Upsilon} \hat{B}\right|_{M}-\left.B\right|_{M}=S(n) \Upsilon$ as desired.

In comparing (4.9) with (2.5), note that $k_{n} S(n)$ is a pseudodifferential operator on $M$ of order $n$, whose principal symbol, by (4.4), is equal to that of $\Delta_{h}^{n / 2}$. A. Chang and J. Qing have pointed out to us that if $n=3$ and the Poincaré metric $g$ is Einstein, then the $Q$-curvature (4.8) agrees with a multiple 
of the curvature quantity $T$ defined in $[\mathrm{CQ}]$ for a suitably chosen metric which is conformal to $g$ and which extends smoothly to the boundary. Similarly, the scattering matrix $S(3)$ for such an Einstein Poincaré metric agrees with a multiple of the boundary operator they call $P_{3}$ when applied to the solution of an appropriately chosen elliptic boundary problem.

Since $S(n)$ is self-adjoint and $S(n) 1=0$, from (4.9) it follows that $\int_{M} Q d v_{h}$ is independent of the choice of $h$ in the conformal class. For the renormalized volume we have the following analogue of Theorem 3.2.

Theorem 4.3. If $n$ is odd and $g$ is a Poincaré metric with conformal infinity $[h]$, then the renormalized volume $V$ in (2.7) is given by

$$
k_{n} V=\int_{M} Q d v_{h}
$$

Proof. Green's identity gives

$$
n \operatorname{vol}_{g}\{x>\epsilon\}=\int_{x>\epsilon} \Delta_{g} U d v_{g}=\epsilon^{1-n} \int_{x=\epsilon} \partial_{x} U d v_{h_{\epsilon}} .
$$

Evaluation of the constant term in the expansion after substituting (4.1) and (2.6) gives $V=\left.\int_{M} B\right|_{M} d v_{h}$ as desired.

\section{References}

[A1] M. Anderson, $L^{2}$ curvature and volume renormalization for AHE metrics on 4manifolds, Math. Res. Lett. 8 (2001), no. 1-2, 171-188.

[A2] - Einstein metrics with prescribed conformal infinity on 4-manifolds, preprint: math.DG/0105243

[B] T. Branson, Sharp inequalities, the functional determinant, and the complementary series, Trans. Amer. Math. Soc. 347 (1995), no. 10, 3671-3742.

$[\mathrm{B} \varnothing] \quad$ T. Branson and B. Ørsted, Explicit functional determinants in four dimensions, Proc. Amer. Math. Soc. 113 (1991), no. 3, 669-682.

[CQ] S.-Y. A. Chang and J. Qing, The zeta functional determinants on manifolds with boundary. I, II. J. Func. Anal. 147 (1997), no. 2, 327-362, 363-399.

[CY] S.-Y. A. Chang and P. C. Yang, Partial differential equations related to the GaussBonnet-Chern integrand on 4-manifolds, The 2000 Barrett Lectures, Univ. of Tennessee, chapter in a volume in the series "Progress in Nonlinear Differential Equations and their Applications," Birkhäuser, to appear.

[FG] C. Fefferman and C. R. Graham, Conformal invariants, The mathematical heritage of Élie Cartan (Lyon, 1984), Astérisque 1985, Numero Hors Serie, 95-116.

[GP] A. R. Gover and L. J. Peterson, Conformally invariant powers of the Laplacian, Qcurvature and tractor calculus, preprint: math-ph/0201030

[G] C. R. Graham, Volume and area renormalizations for conformally compact Einstein metrics, Rend. Circ. Mat. Palermo, (2) Suppl. No. 63 (2000), 31-42.

[GJMS] C. R. Graham, R. Jenne, L. J. Mason, and G. A. J. Sparling, Conformally invariant powers of the Laplacian. I. Existence, J. London Math. Soc. (2) 46 (1992), no. 3, $557-565$.

[GL] C. R. Graham and J. M. Lee, Einstein metrics with prescribed conformal infinity on the ball, Adv. Math. 87 (1991), no. 2, 186-225.

[GW] C. R. Graham and E. Witten, Conformal anomaly of submanifold observables in AdS/CFT correspondence, Nucl. Phys. B 546 (1999), no. 1-2, 52-64; hep-th/9901021 
[GZ] C. R. Graham and M. Zworski Scattering matrix in conformal geometry, preprint: math.DG/0109089

[HS] M. Henningson and K. Skenderis, The holographic Weyl anomaly, J. High Ener. Phys. 07 (1998), 023; hep-th/9806087; Holography and the Weyl anomaly, hep-th/9812032

[JS] M. Joshi and A. Sá Barreto, Inverse scattering on asymptotically hyperbolic manifolds, Acta Math. 184 (2000), no. 1, 41-86.

[Ma1] R. Mazzeo, The Hodge cohomology of a conformally compact metric, J. Differential Geom. 28 (1988), no. 2, 309-339.

[Ma2] Unique continuation at infinity and embedded eigenvalues for asymptotically hyperbolic manifolds, Am. J. Math. 113 (1991), no. 1, 25-45.

[MM] R. Mazzeo and R. B. Melrose, Meromorphic extension of the resolvent on complete spaces with asymptotically constant negative curvature, J. Funct. Anal. 75 (1987), no. 2, 260-310.

[Me] R. B. Melrose, Geometric Scattering Theory, Stanford Lectures, Cambridge University Press, Cambridge, 1995.

[Pa] S. Paneitz, A quartic conformally covariant differential operator for arbitrary pseudo-Riemannian manifolds, preprint, 1983.

[PP] S. J. Patterson and P. A. Perry, The divisor of Selberg's zeta function for Kleinian groups. Appendix A by Charles Epstein. Duke Math. J. 106 (2001), no. 2, 321-390.

[W] E. Witten, Anti-de Sitter space and holography, Adv. Theor. Math. Phys. 2 (1998), no. 2, 253-290; hep-th/9802150

Department of Mathematics, Princeton University, Princeton, NJ 08544, U.S.A.

E-mail address: cf@math.princeton.edu

Department of Mathematics, University of Washington, Box 354350, Seattle, WA 98195, U.S.A.

E-mail address: robin@math.washington.edu 